\title{
A PROOF OF AN OPEN PROBLEM OF YUSUKE NISHIZAWA FOR A POWER-EXPONENTIAL FUNCTION
}

\section{Branko Malešević, Tatjana Lutovac And Bojan BanjaC}

Abstract. This paper presents a proof of the following conjecture, stated by Yusuke Nishizawa in [Appl. Math. Comput. 269, (2015), 146-154.]: for $0<x<\pi / 2$ the inequality $\frac{\sin x}{x}>$ $\left(\frac{2}{\pi}+\frac{\pi-2}{\pi^{3}}\left(\pi^{2}-4 x^{2}\right)\right)^{\theta(x)}$ holds, where $\theta(x)=-\frac{\left(\pi^{3}-24 \pi+48\right) x^{3}}{3(\pi-2) \pi^{3}}+\frac{\pi^{3}}{24(\pi-2)}$.

Mathematics subject classification (2010): 26D05, 41A10.

Keywords and phrases: Mixed logarithmic-trigonometric polynomial functions and inequalities.

\section{REFERENCES}

[1] Y. NishizawA, Sharpening of Jordan's type and Shafer-Fink's type inequalities with exponential approximations, Appl. Math. Comput. 269 (2015), 146-154.

[2] D. S. Mitrinović, Analytic Inequalities, Springer-Verlag, 1970.

[3] G. Milovanović, M. Rassias (ed.), Analytic Number Theory, Approximation Theory and Special Functions, Springer 2014, Chapter: G. D. Anderson, M. Vuorinen, X. Zhang, Topics in Special Functions III, 297-345.

[4] C. MoRTICI, The natural approach of Wilker-Cusa-Huygens inequalities, Math. Inequal. Appl. 14: 3 (2011), 535-541.

[5] C.-P. Chen, W.-S. Cheung, Sharp Cusa and Becker-Stark inequalities, J. Inequal. Appl. 2011: 136 (2011), 1-6.

[6] Z.-H. YANG, New sharp Jordan type inequalities and their applications, Gulf. J. Math. 2: 1 (2014), $1-10$.

[7] B. A. Bhayo, J. SANDor, On certain old and new trigonometric and hyperbolic inequalities, Anal. Math. 41: 3 (2015), 3-15.

[8] C.-P. Chen, J. SAndor, Sharp inequalities for trigonometric and hyperbolic functions, J. Math. Inequal. 9: 1 (2015), 203-217.

[9] Y. NishizAWA, Sharp Becker-Stark's type inequalities with power exponential functions, J. Inequal. Appl. 2015: 402 (2015), 1-11.

[10] M. MiYAGI, Y. NishiZAWA, A stronger inequality of Cirtoaje's one with power-exponential functions, J. Nonlinear Sci. Appl. 8 (2015), 224-230.

[11] Z.-H. YANG, Y.-M. CHU, A Sharp double inequality involving trigonometric functions and its applications, J. Math. Inequal. 10: 2 (2016), 423-432.

[12] B. BANJAC, M. MAKRAGić, B. MALEŠEvić, Some notes on a method for proving inequalities by computer, Results Math. 69: 1 (2016), 161-176.

[13] M. Nenezić, B. MALEŠEvić, C. MoRTICI, Accurate approximations of some expressions involving trigonometric functions, Appl. Math. Comput. 283 (2016), 299-315.

[14] B. Malešević, M. Makragić, A Method for Proving Some Inequalities on Mixed Trigonometric Polynomial Functions, J. Math. Inequal. 10: 3 (2016), 849-876.

[15] B. MALEŠEvić, B. BANJAC, I. Jovović, A proof of two conjectures of Chao-Ping Chen for inverse trigonometric functions, J. Math. Inequal. 11: 3 (2017), 151-162. 